\title{
POLYNOMIALS IN TOPOLOGICAL FIELDS
}

\author{
IRVING KAPLANSKY
}

1. Introduction. Let $F$ be a real closed field in the sense of ArtinSchreier $[1,8],{ }^{1}$ and $f(x)$ a polynomial with coefficients in $F$. It is known that $f(x)$ attains a maximum and minimum in any interval $a \leqq x \leqq b$. Recently, Habicht [4] has begun the study of polynomials in several variables over a real closed field and proved the following theorem: if $f\left(x_{1}, \cdots, x_{n}\right)$ is positive for $-m \leqq x_{i} \leqq m$, then $f$ has a positive lower bound in this region. An equivalent statement is that $f$ maps the region into a closed subset of $F$, where we give $F$ its order topology (the open intervals are a base for the open sets). This latter formulation suggests possible extensions of Habicht's theorem to more general topological fields.

In this note we shall examine such extensions. In $\$ 3$ we obtain quite complete results for polynomials in one variable over fields of "type V" (\$2). But simple examples show that for two or more variables the situation is more complicated. We do however obtain a result (Theorem 4) which immediately implies Habicht's theorem and which is valid for a somewhat wider class of topological fields than real closed fields in their order topology. A further result for polynomials in two variables appears in Theorem 3 .

2. Fields of type $V$. We begin by recalling some definitions. In a topological ring $A$ we call a set $B$ right bounded if for any neighborhood $U$ of 0 there exists a neighborhood $V$ of 0 such that $B V \subset U$. Left boundedness is analogously defined and a set is bounded if it is both right and left bounded. We denote by $A_{n}$ the $n$-dimensional vector space over $A$ (Cartesian product topology), and a subset of $A_{n}$ is bounded if it is bounded coordinate-wise. Any compact set is bounded, and in many arguments such as the following, bounded sets behave somewhat like compact ones.

LEMMA 1. Let $f, g$ be functions defined on a subset $S$ of $A_{n}$ and taking values in $A$. Suppose $f, g$ are bounded and uniformly continuous on $S$. Then $f+g$ and $f g$ are bounded and uniformly continuous on $S$.

The proof is obvious except perhaps for the uniform continuity of $f g$, which depends upon iterated use of the identity

Received by the editors January 6, 1948.

1 Numbers in brackets refer to the bibliography at the end of the paper. 


$$
a b-c d=a(b-d)+(a-c) d .
$$

By repeated application of Lemma 1, we obtain the following corollary.

Lemma 2. A polynomial in $n$ variables is bounded and uniformly continuous on a bounded subset of $A_{n}$.

We shall say that a topological division ring $F$ is of type $V$ if whenever a set $B \in F$ is bounded away from 0 (that is, disjoint from a neighborhood of 0 ), then $B^{-1}$ is bounded. ${ }^{2}$ Except for the omission of any countability restriction, this is the definition given in [6]. Most of the results of [6] are valid without the countability assumption. ${ }^{3}$ In particular [6, Lemma 5]: the completion of a division ring of type $V$ is a division ring of type $V$.

It is easy to verify that the following constitute examples of rings of type $V$ : (a) discrete division rings, (b) division rings with a valuation, (c) ordered division rings in their order topology. It is to be observed that in (a) all sets are bounded, while in (b) and (c) boundedness coincides with its ordinary meaning. Any non-completable division ring is necessarily not of type $V$ : for example, the rationals in the 6-adic topology.

In the sequel we shall require Theorem 11 of [6]. Restated in the Cartan-Bourbaki language of filters, ${ }^{4}$ it reads as follows. (We have simplified by using a fixed polynomial instead of a variable one; only this special case is needed in the present paper.)

Lemma 3. Let $\left\{A_{i}\right\}$ be an ultrafilter in a topological field $F$ of type $V$, and suppose there exists a nonconstant polynomial $f(x)$ with coeffcients in $F$ such that $\left\{f\left(A_{i}\right)\right\}$ is the base of a Cauchy filter. Then $\left\{A_{i}\right\}$ is also Cauchy.

We shall also make use of the following lemma.

Lemma 4. Let $F$ be a topological division ring of type $V$, and $A, B$ subsets of $F$. If $A, B$ are bounded away from 0 , so is $A B$. If $B$ is bounded and $A B$ is bounded away from 0 , then $A$ is bounded away from 0 .

Proof. If $A, B$ are bounded from 0 , then $A^{-1}, B^{-1}$ are bounded and

${ }^{2}$ This axiom also occurs in Bourbaki [3, chap. III, p. 57, Ex. 13] where it is labelled $\mathrm{KT}_{a}$. The only overlapping with [6] is in part (b) of this exercise, which is to show that the completion of a division ring satisfying $\mathrm{KT}_{a}$ is a division ring. I regret having missed this reference till after [6] was published.

8 This does not apply to the main result (Theorem 9) whose proof used the category theorem for complete metric spaces.

${ }^{4} \mathrm{Cf}$. [3] for the relevant definitions. 
so is $B^{-1} A^{-1}=(A B)^{-1}$. Hence $A B$ is bounded from 0 . (A set whose inverses are bounded must be bounded from 0 ; this holds in any topological ring.) Again if $A B$ is bounded from 0 and $B$ is bounded, then $B \cdot(A B)^{-1} \supseteq A^{-1}$ is bounded, whence $A$ is bounded from 0 .

3. One variable. We begin with a result which follows readily from Lemma 3.

THEOREM 1. Let $F$ be a topological field of type $V$, and suppose $F$ is algebraically closed in its completion $G$ (any element of $G$ algebraic over $F$ is already in $F)$. Let $f(x)$ be a polynomial with coefficients in $F$. Then the mapping $x \rightarrow f(x)$ is closed, that is, it sends closed sets into closed sets.

Proof. Let $B$ be a closed set in $F$, and suppose $b$ is in the closure of $f(B)$. Then we can find $\left\{X_{i}\right\}$, a base of a filter approaching $b$, with each $X_{i}$ in $f(B)$. We write $X_{i}=f\left(A_{i}\right)$, with $A_{i} \subset B$. Then $\left\{A_{i}\right\}$ is the base of a filter; we refine it to an ultrafilter which by Lemma 3 is Cauchy, and hence approaches an element $a$ in $G$. Then $f(a)=b$, whence by hypothesis $a \in F, a \in B$. Hence $b \in f(B)$ and $f(B)$ is closed.

REMARK 1 . The hypothesis that $F$ is algebraically closed in $G$ is of course satisfied if $F$ is algebraically closed or if it is complete; the condition is closely related to Ostrowski's relative completeness which says that $F$ is separably algebraically closed in $G[7$, p. 316]. A real closed field in its order topology is algebraically closed in its completion since, as is well known, the completion is again formally real (and even real closed). The necessity of some such condition is shown by an example like $x^{2}-2$ over the rationals.

One can make a partial extension of Theorem 1 to rational functions.

THEOREM 2. Let $F$ be a topological field of type $V$ algebraically closed in its completion, and $S$ a bounded closed subset of $F$. Then for any rational function $f(x)$ with coefficients in $F, f(S)$ is closed. ${ }^{5}$ If $f \neq \infty$ on $S, f(S)$ is also bounded.

Proof. Let $b$ be in the closure of $f(S)$. Write $f=g / h, k=g-b h$, where $g, h, k$ are polynomials. By Lemma $2, h(S)$ is bounded, and hence $1 / h(S)$ is bounded from 0 . We cannot have $k(S)$ bounded from 0 for then by Lemma $4, k / h=f-b$ would be bounded from 0 on $S$. Hence by Theorem $1, k(a)=0$ for $a \in S$. Necessarily $h(a) \neq 0$, for other-

${ }^{5}$ By $f(S)$ we mean the range of $f$, excluding the points where $f=\infty$. It would also be possible to adjoin a formal point at infinity, making its neighborhoods the complements of bounded sets. The theorem is also true with this interpretation. 
wise $g$ and $h$ would have the common factor $x-a$. Thus $f(a)=b$, and $f(S)$ is closed.

If $h \neq 0$ on $S$, then, by Theorem $1, h(S)$ is bounded from $0,1 / h(S)$ is bounded, and $f(S)$ is bounded.

REMARK 2. That Theorem 2 fails if $S$ is unbounded is evident from an example like $1 /\left(x^{2}+1\right)$ over the reals.

4. Several variables. Let $f(x, y)$ be a polynomial with coefficients in $F$. We consider the mapping $(x, y) \rightarrow f(x, y)$ of $F_{2}$ into $F$. If $F$ is locally compact, the mapping sends bounded closed (=compact) sets into closed sets. But it would seem that nothing short of local compactness will suffice to get this result.

Example 1. Completeness does not suffice. Let $F$ be the field of formal power series in a variable $t$ over a field containing infinitely many elements $a_{i}$. We topologize $F$ in the usual way, making $t^{i}$ approach 0 . Then the set $\left(a_{i}+t^{i},-a_{i}\right)$ is bounded and closed, but is mapped by $x+y$ into the nonclosed set $\left\{t^{i}\right\}$.

EXAMPLE 2. It does not help if the completion of $F$ is locally compact. In the field of real algebraic numbers, pick null sequences $a_{i}, b_{i}$ such that $a_{i}+b_{i} \neq 0$, and $\pi+a_{i},-\pi+b_{i}$ are rational. Then $x+y$ maps the closed bounded set $\left(\pi+a_{i},-\pi+b_{i}\right)$ into a nonclosed set.

These examples indicate that for $f(S)$ to be bounded away from 0 it does not suffice to have $S$ closed and disjoint from the manifold $Z(f)$ consisting of all points where $f=0$. A stronger condition is that $S$ be disjoint from a uniform neighborhood of $Z(f)$, by which we mean that there exists a neighborhood $U$ of 0 such that $S$ is disjoint from $Z(f)+U$. For two variables over an algebraically closed field this does suffice, and moreover $S$ need not be bounded.

THEOREM 3. Let $F$ be an algebraically closed field of type $V, f(x, y)$ a polynomial with coefficients in $F$, and $Z(f)$ the manifold $f=0$. Let $S$ be a set disjoint from a uniform neighborhood of $Z(f)$. Then $f(S)$ is bounded away from 0 .

Proof. Write $f(x, y)=a(y) x^{m}+\cdots=b(x) y^{n}+\cdots$ Let $\alpha_{i}, \beta_{j}$ denote the roots of $a, b$ respectively (either or both of these sets may be void in case $a$ or $b$ is a constant). If the point $\left(\beta_{j}, \alpha_{i}\right)$ is in $Z(f)$, then by hypothesis $S$ is disjoint from a neighborhood of it. If $\left(\beta_{j}, \alpha_{i}\right)$ is not in $Z(f)$, then there is a neighborhood of it in which $f$ is bounded from 0 . In either event we thus reduce the problem to a portion of $S$, say $S_{1}$, disjoint from a neighborhood of the points $\left(\beta_{j}, \alpha_{i}\right)$. We may decompose $S_{1}$ into a portion $S_{2}$ where $y-\alpha_{i}$ is bounded from 0 and a portion $S_{3}$ where $x-\beta_{j}$ is bounded from 0 (the sets $S_{2}, S_{3}$ may of 
course overlap). It will suffice to prove that $f$ is bounded from 0 on $S_{2}$. By Theorem 1 , or by a direct application of Lemma $4, a(y)$ is bounded from 0 on $S_{2}$. Now for any fixed $y$ write

$$
f(x, y)=a(y)\left(x-z_{1}\right) \cdots\left(x-z_{m}\right)
$$

where $z_{1}, \cdots, z_{m}$ are the roots of $f(x, y)=0$, so that $\left(z_{i}, y\right) \in Z(f)$. For $(x, y)$ in $S$ it follows from our hypothesis that $x-z_{i}$ is bounded from 0 . By Lemma $4, f$ is bounded from 0 on $S_{2}$.

REMARK 3. I have not determined whether Theorem 3 holds for three or more variables. Algebraic closure however is necessary: take $f=x^{2}+(x y-1)^{2}$ over the reals. Here $Z(f)$ is void, yet $f \rightarrow 0$. One may explain this failure by the necessity of considering the whole complex manifold $f=0$. However if we assume that $S$ is bounded and $F$ real closed, then a result can be obtained just by consideration of the real manifold, and we prove it for any number of variables. The proof is independent of the preceding results.

THEOREM 4. Let $F$ be a topological real closed field such that $F(i)$, in its Cartesian product topology, is of type V. ${ }^{6}$ Let $f\left(x_{1}, \cdots, x_{n}\right)$ be a polynomial with coefficients in $F$, and $Z(f) \subset F_{n}$ the manifold $f=0$. Let $S$ be a bounded set in $F_{n}$, disjoint from a uniform neighborhood of $Z(f)$. Then $f(S)$ is bounded away from 0 .

Proof. Let us write $x$ for $x_{1}, y$ for $\left(x_{2}, \cdots, x_{n}\right)$ and $f(x, y)=a(y) x^{m}$ $+\cdots$. We shall first prove the theorem under the assumption that $a(y)$ is bounded from 0 on $S$. Let $z_{1}, \cdots, z_{m}$ denote the roots of $f(x, y)=0$. Following Habicht's fundamental device, we form the $M=m(m-1) / 2$ symmetric functions in the quantities $\left(z_{i}-z_{j}\right)^{2}(i<j)$. After multiplication by suitable powers of $a(y)$ these become polynomials $G_{i}(y)(i=1, \cdots, M)$. We choose the numbering so that $G_{1}$ corresponds to the product, $G_{M}$ to the sum of the roots. Let $Z_{j}$ denote the manifold $G_{1}=G_{2}=\cdots=G_{j}=0$, and $Z_{0}$ the entire space. We may embed $S$ in a sequence of sets $S=S_{0} \subset S_{1} \subset \cdots \subset S_{M}$ such that each $S_{i}$ contains a uniform neighborhood of $S_{i-1}$, and we may suppose that $S_{M}$ is still bounded and uniformly bounded from $Z(f)$, while $a(y)$ is still bounded from 0 on $S_{M}$ (the latter is possible by Lemma 2). Set $T_{i}=S_{i} \cap Z_{i}$. We shall prove: (1) $f$ is bounded from 0 on $T_{M}$, (2) if $f$ is bounded from 0 on $T_{i+1}$, it is likewise bounded from 0 on $T_{i}$. Since $T_{0}=S_{0}=S$, this will prove the desired result.

(1) On $Z_{M}$, all the $z_{i}$ are equal and hence real. It follows from

${ }^{6}$ This condition is equivalent to the assertion that $1+x^{2}$ is bounded from 0 in $F$, and the latter statement of course holds for any ordered field in its order topology. Also, by [6, Theorem 12], $F(i)$ will be of type $V$ if $F$ is complete. 
hypothesis that on $T_{M}, x-z_{i}$ is bounded from 0 . By Lemma 4 so is $f(x, y)$, which is given by (1).

(2) We suppose $f$ bounded from 0 on $T_{i+1}$ and select a neighborhood $V$ of 0 small enough so that $f$ is still bounded from 0 on $T_{i+1}+V$. Thus it remains to prove that $f$ is bounded from 0 on $T_{i}$ with $T_{i} \cap\left(T_{i+1}+V\right)$ deleted; call this diminished set $T_{i}^{*}$. We assert that $T_{i}^{*}$ is disjoint from a uniform neighborhood of $Z_{i+1}$. For by construction $T_{i}^{*}$ is disjoint from a uniform neighborhood of the complement of $T_{i+1}$; and within $T_{i+1}$ we have in the formation of $T_{i}^{*}$ deleted all points within $V$ of $Z_{i+1}$. Now $Z_{i+1}$ is the zero manifold of $G_{1}^{2}+\ldots$ $+G_{i+1}^{2}$. By an induction on $n$ (the number of variables) we can assert that on $T_{i}^{*}, G_{1}^{2}+\cdots+G_{i+1}^{2}$ is bounded from 0 . Since $G_{1}$ to $G_{i}$ vanish on $T_{i}^{*}$, we have that $G_{i}$ is bounded from 0 . Now $G_{i+1}$ is (except for a power of $a(y)$ which does not affect the argument) precisely the product of all nonvanishing terms among $\left(z_{i}-z_{j}\right)^{2}$. For this to be bounded from 0 , all these nonvanishing $z_{i}-z_{j}$ must likewise be bounded from 0 (one needs here the fact that the $z$ 's are bounded, an easy consequence of the boundedness of the coefficients of $f$, together with the fact that the leading coefficient is bounded from 0 ). In particular the imaginary parts of the complex roots, if there are any, must be bounded from 0 , since these occur in conjugate pairs. Now we observe that in (1), $x-z_{i}$ is bounded from 0 for real $z_{i}$ by hypothesis, while for complex $z_{i}$, the imaginary part of $x-z_{i}$ is already bounded from 0 . Hence $f$ is bounded from 0 .

We now treat the general case where it is not assumed that $a(y)$ is bounded from 0 . Changing notation we write

$$
\begin{aligned}
f(x, y) & =a_{0}(y) x^{m}+a_{1}(y) x^{m-1}+\cdots+a_{m}(y), \\
h_{i}(y) & =a_{0}^{2}+a_{1}^{2}+\cdots+a_{i}^{2} .
\end{aligned}
$$

We note that $Z\left(h_{m}\right) \subseteq Z(f)$, and so $S$ is disjoint from a uniform neighborhood of $Z\left(h_{m}\right)$. Since $h_{m}$ is independent of $x$, we have by induction on $n$ that $h_{m}$ is bounded from 0 on $S$. Consider now the following proposition which we shall designate as $P_{i}$ : if for a set $T \subseteq S, h_{i}(T)$ is known to be bounded from 0 , then $f(T)$ is bounded from 0 . We know the truth of $P_{0}$ from the first part of the proof. On the other hand $P_{m}$ is the theorem we are trying to prove, for we have seen that $h_{m}(S)$ is bounded from 0 . Hence it will suffice to prove that $P_{i}$ implies $P_{i+1}$. So we suppose $P_{i}$ known and $h_{i+1}(T)$ bounded from 0 , and have to prove $f(T)$ bounded from 0 . Now there is a neighborhood $V$ of 0 such that $a_{i+1} \in V$ implies that $h_{i}(T)$ is bounded from 0 . We delete from $T$ the portion where $a_{i+1} \in V$, for there we know from $P_{i}$ that $f$ is 
bounded from 0 , and study the remaining part, say $T_{1}$. We write

$$
r(x, y)=a_{i+1} x^{m-1-i}+\cdots+a_{m}, \quad k(x, y)=h_{i}+r^{2} .
$$

Evidently $Z(k) \subseteq Z(f)$ and hence $S$ is disjoint from a uniform neighborhood of $Z(k)$. Moreover in $T_{1}$ the leading coefficient of $k$ is bounded from 0 . By the first part of the proof, $k\left(T_{1}\right)$ is bounded from 0 . Now by choosing $h_{i}$ small enough we can make

$$
f-r=a_{0} x^{m}+\cdots+a_{i} x^{m-i}
$$

arbitrarily small in $S$ (this is because $S$ is bounded). Hence there exists a neighborhood $W$ of 0 such that $h_{i} \in W$ implies that $k, r, f$ are all bounded from 0 on $T_{1}$. In that portion of $T_{1}$ where $h_{i}$ is not in $W$ we have again by $P_{i}$ that $f$ is bounded from 0 . This shows that $f$ is bounded from 0 throughout $T$ and proves $P_{i+1}$. This completes the proof of Theorem 4.

TheOREM 5 (НABICHT). Let $F$ be a real closed field in its order topology, and $R$ the region $a_{i} \leqq x \leqq b_{i}(i=1, \cdots, n)$. Then for any rational function $f\left(x_{1}, \cdots, x_{n}\right), f(R)$ is closed.

The deduction of Theorem 5 from Theorem 4 is identical with Habicht's but we give it for completeness. We suppose $f$ is a polynomial; the device used in the proof of Theorem 2 carries out the extension to a rational function. We may suppose $f \neq 0$ on $R$ and are to prove $f(R)$ bounded from 0 . By induction on $n$ we have $f\left(R^{\prime}\right)$ bounded from 0 , where $R^{\prime}$ is the boundary of $R$. This implies that $R^{\prime}$ is disjoint from a uniform neighborhood of $Z(f)$. Then $R$ is likewise disjoint from a uniform neighborhood of $Z(f)$ (at this point we are using very strongly the special properties of an order topology). Quotation of Theorem 4 completes the proof.

REMARK 4. This method of proof will work for more general regions, for example, regions defined inductively by the property that the boundary lies on a finite number of hyperplanes. On the other hand our examples above have shown that we cannot take an arbitrary bounded closed set for $R$. One might hazard the conjecture that a bounded region defined by a finite number of polynomial inequalities will do.

5. A correction. Professor Jacobson has pointed out an error in [6] which I take this opportunity to correct. The proof of Lemma 9 is inconclusive since the possibility that $L$ is commutative is not excluded, and in fact the validity of Lemma 9 as stated appears to be an open question. Lemma 9 should be deleted, and part (a) of the 
proof of Theorem 9 should be replaced by the following remark: if $D$ is a central algebraic division algebra over a field $F$, with the property that all separable subfields of $D$ are finite-dimensional, then $D$ is finite-dimensional. (This is a slight generalization of [5, Theorem 7].)

To prove this we take (Zorn's lemma) a maximal separable subfield $K$ and let $L$ be its centralizer. By hypothesis $K$ is finite-dimensional and hence [2, Theorem 13] the centralizer of $L$ is again $K$. The argument of [5, Lemma 2] is now applicable and shows that $L=K$. By [2, Theorem 13] again, $[D: K]=[K: F]$ is finite and $D$ is finitedimensional.

\section{BIBLIOGRAPHY}

1. E. Artin and O. Schreier, Algebraische Konstruktion reeler Körper, Abh. Math. Sem. Hamburgischen Univ. vol. 5 (1926) pp. 83-115.

2. E. Artin and G. Whaples, The theory of simple rings, Amer. J. Math. vol. 65 (1943) pp. 87-107.

3. N. Bourbaki, Eléments de Mathématique, Livre III. Topologie Générale, Actualités Scientifiques et Industrielles, nos. 858 and 916, Paris, 1940 and 1942.

4. W. Habicht, Ein Existenzsatz über reele definite Polynome, Comment. Math. Helv. vol. 18 (1946) pp. 331-348.

5. N. Jacobson, Structure theory for algebraic algebras of bounded degree, Ann. of Math. vol. 46 (1945) pp. 695-707.

6. I. Kaplansky, Topological methods in valuation theory, Duke Math. J. vol. 14 (1947) pp. 527-541.

7. A. Ostrowski, Untersuchungen zur arithmetischen Theorie der Körper, Math. Zeit. vol. 39 (1935) pp. 269-404. 245.

8. B. L. van der Waerden, Moderne Algebra, 2d ed., Berlin, 1940, vol. 1, pp. 235-

University of Chicago 